\title{
Nanoparticles as mitochondria-targeted photosensitizer in photodynamic therapy of cancer
}

\author{
Mei-Jie Jou ${ }^{1,2 *}$ and Tsung-I Peng ${ }^{2,3}$ \\ ${ }^{1}$ Department of Physiology and Pharmacology, College of Medicine, Chang Gung University, Tao-Yuan, Taiwan \\ ${ }^{2}$ Department of Neurology, Chang Gung Memorial Hospital, Keelung Branch, Keelung, Taiwan \\ ${ }^{3}$ Department of Medicine, Chang Gung University, Tao-Yuan, Taiwan
}

\section{Photodynamic effect of mitochondria-targeted photosensitizers}

Photodynamic therapy (PDT) is a novel and promising treatment for cancer treatment, which involves the administration of photosensitizers coupled with a specific wavelength of laser irradiation. In the presence of oxygen, the PDT with particular photosensitizer causes significant formation of singlet oxygen as well as other reactive oxygen species (ROS), which produce severe peroxidative reaction as a major contribution to light-induced cell damage and death. To achieve the best efficacy of PDT, an ideal photosensitizer obliges good tissue penetration, low dark toxicity (effect of photosensitizer alone) and high extinction coefficient. In addition, subcellular distribution of photosensitizers appears to be critically involved in the potency of PDT [1]. Recently, the mitochondrion has been considered as a novel pharmacological target for clinical application including cancer therapy due to its crucial role involved in arbitrating cell apoptosis. Mitochondrial dysfunction leads not only to the interruption of ATP supply but also to the activation of mitochondrial dysfunctionmediated apoptotic pathway. Key mitochondrial mechanism involved is the opening of the mitochondrial permeability transition pore (MPTP), which results in mitochondrial membrane potential $(\Delta \psi \mathrm{m})$ depolarization, mitochondrial swelling and release of mitochondrial apoptotic lethal proteins to the cytosol. Subsequently, downstream caspases dependent or independent nuclear proteases are activated to
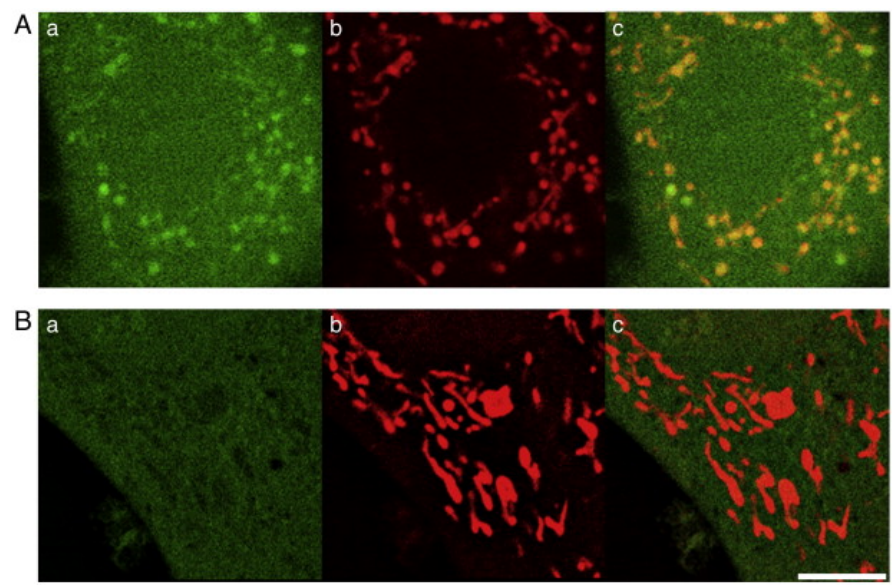

Figure 1. PDE of $488 \mathrm{~nm}$ laser coupled $\mathrm{C}_{3}$-induced mROS "hot spots" and Mito Q-induced "cold spots". A) In the absence of Mito Q. B) In the presence of MitoQ (100 nM). PDE of $\mathrm{C}_{3}(100 \mu \mathrm{M})$ was coupled with $60 \mathrm{~s}$ of $488 \mathrm{~nm}$ laser irradiation. Aa and Ba, DCF images; $\mathrm{Ab}$ and $\mathrm{Bb}$, TMRM image; Ac and Bc merged image of DCF and TMRM. Bar, $5 \mu \mathrm{m}$. cleave nuclear DNA to small fragments. Intriguingly, mitochondriaassociated apoptotic mechanisms, particularly the opening of the MPTP and the release of mitochondrial lethal proteins can be initiated and augmented by mitochondrial ROS (mROS) formation as well as mitochondrial free calcium $\left(\mathrm{mCa}^{2+}\right)$ overload [2-8]. Thus, PDT with an ideal photosensitizer that is able to localize in the mitochondria may significant promote the generation of mROS and then mROSdependent $\mathrm{mCa}^{2+}$ elevation. These two PDT-induced pathological conditions may then interact together to achieve a vicious augmentation of mitochondrial dysfunction and to ensure a potent and successful cell apoptosis for a high efficacy of PDT for tumor eradication.

\section{Mitochondria-targeted nanoparticle photosensitizers: quantum dots (QDs) and carbon sixty (C60)}

The high photosensitizing character of nano-semiconductor QDs provides great therapeutic potential in cancer therapy $[9,10]$. Using laser scanning confocal microscopy we demonstrated QDs localized into the mitochondria. Upon visible light coupled PDE of QDs, mROS-mediated apoptosis was observed. The mitochondrial protector, melatonin, and the mitochondria-targeted antioxidant MitoQ significantly inhibited visible light coupled PDE of QDs-induced apoptosis [11]. Similarly, carbon nanoparticle fullerenes $\left(\mathrm{C}_{60}\right)$ possess also unique photosensitizing character. Recent chemical modifications on the C60 sphere including tris-malonic acid fullerene $\left(\mathrm{C}_{3}\right)$ increases significantly

*Correspondence to: Jou MJ, Department of Physiology and Pharmacology, College of Medicine, Chang Gung University, Tao-Yuan, and Department of Neurology, Chang Gung Memorial Hospital, Keelung Branch, Keelung, Taiwan, E-mail: mjjou@mail.cgu.edu.tw

Special Issue: Nanotechnology: Challenges and Perspectives in Medicine

Dr. Federica Valentini

Department of Sciences and Chemical Technologies

Tor Vergata University

Italy

Maurizio Talamo

Professor

Department of Enterprise Engineering

Italy

Key words: cancer, mitochondria-targeted photosensitizer, nanoparticles, photodynamic therapy

Received: October 19, 2018; Accepted: October 29, 2018; Published: October 31,2018 
its solubility in water and hence its application in disease-based nanobiomedical science $[12,13]$. PDE of $\mathrm{C}_{3}(100 \mathrm{nM})$ coupled with $488 \mathrm{~nm}$ laser induces significant mROS formation and $\Delta \psi \mathrm{m}$ depolarization which causes marked fission and swelling of mitochondria (Figure 1). Mitochondrial hot spots due to significant mROS formation caused significant loss of TMRM indicating depolarized $\Delta_{\psi \mathrm{m}}$ [14]. PDE of visible light coupled mitochondria-targeted nano photosensitizers via enhancing mROS-mediated mitochondrial stress leading to enhanced apoptosis or necrosis. Thus, these nanoparticle-coupled photodynamic therapy may serve as a potential maneuver for clinical treatment of tumors and cancer in the CNS including glioma and astrocytoma.

\section{Acknowledgements}

The authors would like to thank the technical support provided from Microscopy Core Laboratory, Chang Gung Memorial Hospital, Linkou, Taiwan, and Microscopy Center at Chang Gung University, Tao-Yuan, Taiwan. This work was supported by Grants MOST 1062320-B-182-001 and MOST 107-2320-B-182-018 (to Jou); MOST 102-2314-B-182A-061 and MOST 103-2314-B-182A-024-MY3 (to Peng) from the Ministry of Science and Technology, Taiwan.

\section{References}

1. Oleinick NL, Morris RL, Belichenko I (2002) The role of apoptosis in response to photodynamic therapy: what, where, why, and how. Photochem Photobiol Sci 1: 1-21. [Crossref].

2. Jou MJ, Peng TI, Sheu SS 91996) Histamine induces oscillations of mitochondrial free $\mathrm{Ca}^{2+}$ concentration in single cultured rat brain astrocytes. Journal of Physiol 497: 299308.

3. Peng TI, Jou MJ, Sheu SS, Greenamyre JT (1998) Visualization of NMDA receptorinduced mitochondrial calcium accumulation in striatal neurons. Experiment Neurol 149: $1-12$.
4. Jou MJ, Jou SB, Chen HM, Lin CH, Peng TI (2002) Critical role of mitochondrial reactive oxygen species formation in visible laser irradiation-induced apoptosis in rat brain astrocytes (RBA-1). J Biomed Sci 9: 507-516.

5. Jou MJ, Jou SB, Guo MJ, Wu HY, Peng TI (2004) Mitochondrial reactive oxygen species generation and calcium increase induced by visible light in astrocytes. Ann N Y Acad Sci 1011: 45-56.

6. Peng TI, Jou MJ (2004) Mitochondrial swelling and generation of reactive oxygen species induced by photoirradiation are heterogeneously distributed. Ann N Y Acad Sci 1011: 112-122.

7. Peng TI, Chang CJ, Guo MJ, Wang YH, Yu JS, et al. (2005) Mitochondrion-Targeted Photosensitizer Enhances the Photodynamic Effect-Induced Mitochondrial Dysfunction and Apoptosis. Ann N Y Acad Sci 1024: 419-428.

8. Hsiao CW, Peng TI, Peng AC, Reiter RJ, Tanaka M, et al. (2013) Long-term Aß exposure augments $\mathrm{mCa}^{2+}$-independent $\mathrm{mROS}$-mediated depletion of cardiolipin for the shift of a lethal transient mitochondrial permeability transition to its permanent mode in NARP cybrids: a protective targeting of melatonin. J Pineal Res 54: 107-125.

9. Bakalova R, Ohba H, Zhelev Z, Ishikawa M, Baba Y (2004) Quantum dots as photosensitizers? Nat Biotechnol 22: 1360-1361.

10. Samia AC, Chen X, Burda C (2003) Semiconductor quantum dots for photodynamic therapy. J Am Chem Soc 125: 15736-15737.

11. Jou MJ, Su YF (2006) Quantitative investigation of mitochondrial DNA mutationaugmented mitochondrial dysfunction upon oxidative stress, calcium stress and photodynamic effect of quantum nanoparticles. Master thesis, Chang Gung University, Taiwan.

12. Bosi S, Ros TD, Spalluto G, Prato M (2003) Fullerene derivatives: an attractive tool for biological applications. Eur J Med Chem 38: 913-923.

13. Lamparth I, Hirsch A (1994) Water-soluble malonic acid derivatives of C60 with a defined three-dimensional structure. J Chem Soc Chem Cumm 1727-1728.

14. Jou MJ (2008) Pathophysiological and pharmacological implications of mitochondriatargeted reactive oxygen species generation in astrocytes. Adv Drug Deliv Rev 60 : $1512-1526$.

Copyright: (C2018 Mei-Jie J. This is an open-access article distributed under the terms of the Creative Commons Attribution License, which permits unrestricted use, distribution, and reproduction in any medium, provided the original author and source are credited. 